\title{
A Randomized, Double-Blind Pilot Trial of Hydrolyzed Rice Bran versus Placebo for Radioprotective Effect on Acute Gastroenteritis Secondary to Chemoradiotherapy in Patients with Cervical Cancer
}

\author{
Yoshiyuki Itoh, ${ }^{1}$ Mika Mizuno, ${ }^{2}$ Mitsuru Ikeda, ${ }^{3}$ Rie Nakahara, ${ }^{1}$ Seiji Kubota, ${ }^{1}$ Junji Ito, ${ }^{1}$ \\ Tohru Okada, ${ }^{1}$ Mariko Kawamura, ${ }^{1}$ Fumitaka Kikkawa, ${ }^{2}$ and Shinji Naganawa ${ }^{1}$ \\ ${ }^{1}$ Department of Radiology, Nagoya University Graduate School of Medicine, Nagoya, Aichi 4668550, Japan \\ ${ }^{2}$ Department of Obstetrics and Gynecology, Nagoya University Graduate School of Medicine, Nagoya, Aichi 4668550, Japan \\ ${ }^{3}$ Department of Radiological Technology, Nagoya University Graduate School of Medicine, Nagoya, Aichi 4610047, Japan
}

Correspondence should be addressed to Yoshiyuki Itoh; itoh@med.nagoya-u.ac.jp

Received 13 June 2015; Revised 29 August 2015; Accepted 7 September 2015

Academic Editor: Cheppail Ramachandran

Copyright (C) 2015 Yoshiyuki Itoh et al. This is an open access article distributed under the Creative Commons Attribution License, which permits unrestricted use, distribution, and reproduction in any medium, provided the original work is properly cited.

\begin{abstract}
We aimed to evaluate the radioprotective effect of hydrolyzed rice bran (HRB) on acute gastroenteritis due to chemoradiotherapy for treatment of cervical cancer. This placebo-controlled, double-blind study was conducted as an exploratory investigation of the colitis-inhibiting effects of HRB in alleviating acute-phase gastrointestinal side effects of chemoradiotherapy. The study involved 20 patients (10 in the HRB group, 10 in the control group). The patients in the control group underwent the same chemoradiotherapy regimen as those in the HRB group, but they received a placebo instead of HRB. The diarrheal side effect assessment score was lower in the HRB than control group, and a trend toward a reduction in diarrhea symptoms was observed with the oral intake of HRB. Additionally, no significant difference was observed in the administration of intestinal regulators and antidiarrheal agents, but again the assessment score was lower in the HRB than control group, and diarrhea symptoms were alleviated with the oral intake of HRB. A trend toward no need for strong antidiarrheal agents was seen. Although this study was an exploratory clinical trial, the results suggest that HRB may relieve diarrhea, an acute-phase gastrointestinal side effect of chemoradiotherapy.
\end{abstract}

\section{Introduction}

Radical treatment for cervical cancer generally involves surgical treatment or radiation therapy, either alone or in combination. Recent reports have described better treatment outcomes with chemoradiotherapy than with radiation therapy alone [1-4]. Standard radiation therapy for cervical cancer includes brachytherapy with or after external-beam radiation therapy (EBRT). Because a large part of the pelvic region is irradiated during EBRT, much of the healthy tissue of the small intestine, colon, and rectum is included in the radiation field. The severity of side effects (adverse events) in the acute phase depends on the volume of the irradiation field [5]. Gastrointestinal side effects of radiotherapy in the acute phase include abdominal pain and diarrhea secondary to radiation enteritis. Acute-phase mucosal symptoms involving the urinary system include frequent urination and blood in the urine secondary to radiation cystitis and urethritis. All of these adverse effects decrease patients' quality of life during treatment. When chemotherapy is combined with radiation for added potency, these acute-phase symptoms increase in severity $[6,7]$. Intensity-modulated radiation therapy, a new radiotherapy technique that attempts to reduce the dose of radiation to normal tissue, is one means of reducing gastrointestinal symptoms $[5,7]$.

In current clinical practice, diarrhea accompanying radiation therapy is symptomatically treated by administration of intestinal regulators, antidiarrheal agents, and similar medications $[8,9]$. Therefore, an effective therapy to alleviate the inflammatory effects that occur with radiation therapy is 
needed. Hydrolyzed rice bran (HRB) (Daiwa Pharmaceutical Co., Ltd., Tokyo, Japan) is a processed food, the basic ingredient of which is water-soluble rice bran fiber. Trials of its effectiveness and safety have been conducted in animals and humans. HRB exhibited a significant inflammationinhibitory effect in an experimental murine model of dextran sulfate-induced colitis when compared with the control group (unpublished data). Another study found that HRB may have an anti-inflammatory effect by inhibition of mast cell degranulation and cytokine production in bone marrowderived mast cells [10]. Furthermore, an investigation of the effects of HRB on the prevalence of the common cold syndrome and severity of cold symptoms in older patients was performed [11]. Although no significant difference was seen in the prevalence of the cold syndrome between the HRB and control groups, the severity of symptoms was significantly milder in the HRB than control group.

Therefore, to verify the colitis-inhibiting action of HRB, we conducted an exploratory investigation of the ability of HRB to alleviate acute-phase gastrointestinal side effects of chemoradiotherapy in patients with cervical cancer. In animals HRB enhances NK activity of aged mice and may be useful for enhancing NK function in humans $[12,13]$. We also evaluated the changes in natural killer $(\mathrm{NK})$ cell activity as an immune-enhancing action with the combined use of HRB.

\section{Patients and Methods}

2.1. Patients. All patients in this trial underwent chemoradiotherapy for cervical cancer. Patients with primary squamous cell carcinoma, adenocarcinoma, or adenosquamous carcinoma located in the cervix were included. Patients with small cell carcinoma or sarcoma were excluded. The present study was approved by the investigational review board of our hospital (number 993), and written informed consent was obtained from all patients included in this study. This clinical trial is registered in the UMIN Clinical Trials Registry (UMIN000004350).

The inclusion criteria were as follows: (i) age of $\geq 20$ to $<75$ years at the time of providing informed consent; (ii) cervical cancer with the intent for chemoradiotherapy; (iii) adequately maintained major organ function (bone marrow, liver, and kidneys) and laboratory parameters within the following ranges: white blood cell count of $>3500 / \mathrm{mm}^{3}$, absolute neutrophil count of $>1500 / \mathrm{mm}^{3}$, hemoglobin Alc level of $\geq 10.0 \mathrm{~g} / \mathrm{dL}$, platelet count of $\geq 100,000 / \mathrm{mm}^{3}$, total bilirubin level of $\leq 1.5 \mathrm{mg} / \mathrm{dL}$, aspartate transaminase and alanine transaminase levels of $<80 \mathrm{IU} / \mathrm{L}$, serum creatinine level of $<1.5 \mathrm{mg} / \mathrm{dL}$, and creatinine clearance rate of $\geq 60 \mathrm{~mL} / \mathrm{min}$ (Cockcroft-Gault formula or $24 \mathrm{~h}$ creatinine clearance); and (iv) having received an explanation of the purpose and methods of this trial and having provided written consent prior to the start of the trial.

The exclusion criteria were as follows: (i) undergoing surgical treatment; (ii) undergoing a nonsurgical treatment thought to affect treatment with HRB and its outcome; (iii) presence of a drug allergy; (iv) known or possible pregnancy, desire to become pregnant, or currently breastfeeding; and (v) other conditions that the principal investigator or a coresearcher thought they might make an individual unsuitable for this study.

The study sample comprised 20 patients (10 in the HRB group, 10 in the control group). This sample size was based on the fact that this was an exploratory study and did not have enough statistical power. This was a pilot study to calculate the efficacy rate of $\mathrm{HRB}$, which is necessary to estimate sample sizes for later studies, and to collect safety information.

2.2. Methods. This was a single-institution, randomized, placebo-controlled, double-blind study conducted at Nagoya University Hospital. The HRB tested in this trial was a processed food, the main component of which was a partial hydrolysate of rice bran produced by the action of polysaccharide hydrolytic enzymes of the supernatant of a mycelium culture of Lentinula edodes in water-soluble rice bran fiber. The main component of the placebo food substance given to the control group was dextrin, a watersoluble polysaccharide, and it did not include HRB. Both food substances were given in the form of granules sealed on three sides in an aluminum foil processed film of the same shape and were indistinguishable by appearance. This study coordinator encodes two food substances with matching random numbers, and patients are randomly assigned to the control or HRB group. Neither patient, nor their doctor knows until the end of this trial. Patients who gave written informed consent were randomly assigned to either the HRB group or the control group.

In the HRB group, three packets of the HRB (1 g of HRB per packet) were taken orally three times per day. In the control group, three packets of the placebo food were taken orally three times per day. The HRB or placebo was consumed before the start of chemoradiotherapy (up to 1 week before) and it was taken every day during receiving radiation therapy. Its use of each drug has been also stopped simultaneously with EBRT end.

The survey measures were patient characteristics, clinical laboratory test parameters, and gastrointestinal symptoms. The patient characteristics included diagnosis, birthdate, age, sex, body weight, inpatient/outpatient status, medical history, and complications. The clinical laboratory tests included blood tests (erythrocyte count, hemoglobin level, hematocrit, white blood cell count, and platelet count), serum chemistry tests (levels of aspartate transaminase, alanine transaminase, gamma-glutamyl transpeptidase, alkaline phosphatase, total bilirubin, total protein, total cholesterol, lactate dehydrogenase, triglycerides, uric acid, blood urea nitrogen, and creatinine), and NK cell activity. The gastrointestinal symptoms evaluated were diarrhea, nausea, vomiting, and lack of appetite; both their frequency and severity were assessed.

The time course of the study is shown in Table 1.

The primary endpoint of this study was the frequency and severity of diarrhea symptoms, and the secondary endpoints were the frequency and severity of gastrointestinal symptoms other than diarrhea (nausea, vomiting, and loss of appetite) and NK cell activity. NK cell activity was compared between the two study groups before intake of the HRB or placebo and 
TABLE 1: Time course of the study.

\begin{tabular}{|c|c|c|c|c|c|c|}
\hline \multirow{2}{*}{ Items } & \multirow{2}{*}{ Before uptake } & \multicolumn{4}{|c|}{ Treatment } & \multirow{2}{*}{$\begin{array}{l}\text { Stop of } \\
\text { this study }\end{array}$} \\
\hline & & Before & Start & 3 weeks & End & \\
\hline Informed consent & $\bigcirc$ & & & & & \\
\hline $\begin{array}{l}\text { Subjective or objective } \\
\text { complaints }\end{array}$ & $\bigcirc$ & & $\bigcirc$ & $\bigcirc$ & $\bigcirc$ & $\bigcirc$ \\
\hline Clinical laboratory tests & $\bigcirc$ & & & & $\bigcirc$ & $\bigcirc$ \\
\hline NK cell activity & $\bigcirc$ & & & & $\bigcirc$ & \\
\hline Test food (HRB or placebo food) & & & & & & \\
\hline Chemoradiotherapy & & & 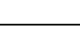 & & & \\
\hline Adverse events & & $\leftarrow$ & & & & \\
\hline
\end{tabular}

at the completion of radiation therapy. All symptoms were scored numerically according to the following criteria.

Diarrhea. 0, no symptoms; 1 , mild symptoms (bowel movements up to 3 times more frequent than normal; passage of stool slightly more frequent than normal); 2 , moderate symptoms (bowel 4 to 6 times more frequent than normal; $<24$-hour intravenous drip is ordered; passage of stool moderately more frequent than normal, but this does not interfere with daily life); and 3, severe symptoms (bowel movements $\geq 7$ times more frequent than normal; incontinence; $\geq 24$ hour intravenous drip is ordered, hospitalization necessary; passage of stool is markedly more frequent than normal and interferes with daily life).

Administration of Intestinal Regulators and Antidiarrheal Agents. 0, not administered; 1, administration of agents containing lactic acid bacilli or bifidobacteria (Lactobacillus casei or Clostridium butyricum); 2, administration of agents containing albumin tannate or berberine chloride; and 3, administration of agents containing loperamide hydrochloride.

Nausea. 0 , no symptoms; 1 , mild symptoms (loss of appetite without change in eating habits); 2, moderate symptoms (decreased oral intake without marked loss of weight, dehydration symptoms, or undernourishment; <24-hour intravenous drip is ordered); and 3 , severe symptoms (poor intake of calories and water by mouth; $\geq 24$-hour intravenous drip, enteral feeding, or total parenteral nutrition is ordered).

Vomiting. 0, no symptoms; 1, mild symptoms (once in 24 hours); 2 , moderate symptoms (2-5 times in 24 hours; <24hour intravenous drip is ordered); and 3, severe symptoms ( $\geq 6$ times in 24 hours; $\geq 24$-hour intravenous drip or total parenteral nutrition is ordered).

Loss of Appetite. 0, no symptoms; 1, mild symptoms (loss of appetite without change in eating habits); 2 , moderate symptoms (decreased oral intake without marked loss of weight or undernourishment; oral nutrition supplements ordered); and 3 , severe symptoms (decreased oral intake with marked loss of weight or undernourishment; drip infusion, enteral feeding, or total parenteral nutrition is ordered).
2.3. Safety Assessment. Safety assessment was performed by evaluating laboratory values before and upon completion of taking the HRB, and they were graded by the National Cancer Institute scale (Common Terminology Criteria for Adverse Events, v3.0 [12]). Also, grade refers to the severity of the diarrhea according to the CTCAE v3.0, but when there was no adverse event, it was made grade " 0 ." Grade 4 is the adverse event associated with life-threatening consequences and it was excluded from this grading.

2.4. Treatment for Cervical Cancer. Radiation therapy involved a combination of EBRT and brachytherapy. EBRT was performed at $1.8 \mathrm{~Gy}$ once per day (total dose of $50.4 \mathrm{~Gy}$ in 28 fractions). Central shielding was performed upon starting brachytherapy. EBRT was applied at $1.8 \mathrm{~Gy}$ once a day, five times a week (Monday-Friday) (total dose of $50.4 \mathrm{~Gy}$ in 28 fractions). When whole-pelvis radiation was performed, four-field radiation of the anterior-posterior and two lateral portals was used as a rule. The radiotherapy methods used in our institute are detailed elsewhere [13]. EBRT alone was performed in patients who could not undergo brachytherapy for some reason (e.g., the tandem and ovoid instruments could not be inserted because of a markedly narrow vagina; deformation was present due pubic bone or other pelvic fractures, or instruments could not be inserted for some other reason).

The following chemotherapy regimen was performed every 3 weeks: cisplatin at $70 \mathrm{mg} / \mathrm{m}^{2}$ on day 1 and a continuous infusion of 5 -fluorouracil at $700 \mathrm{mg} / \mathrm{m}^{2}$ on days 1 to 4 .

2.5. Statistical Analysis. The differences in the side effect assessment scores between the HRB and control groups were compared with Fisher's exact test. The NK cell activity values and white blood cell counts were analyzed by repeatedmeasures analysis of variance. A $P$ value of $<0.05$ was taken to indicate a statistically significant difference.

\section{Results}

3.1. Study Population. The patients' median age was 47.5 years (range, 30-72 years). The histological types of cervical cancer 


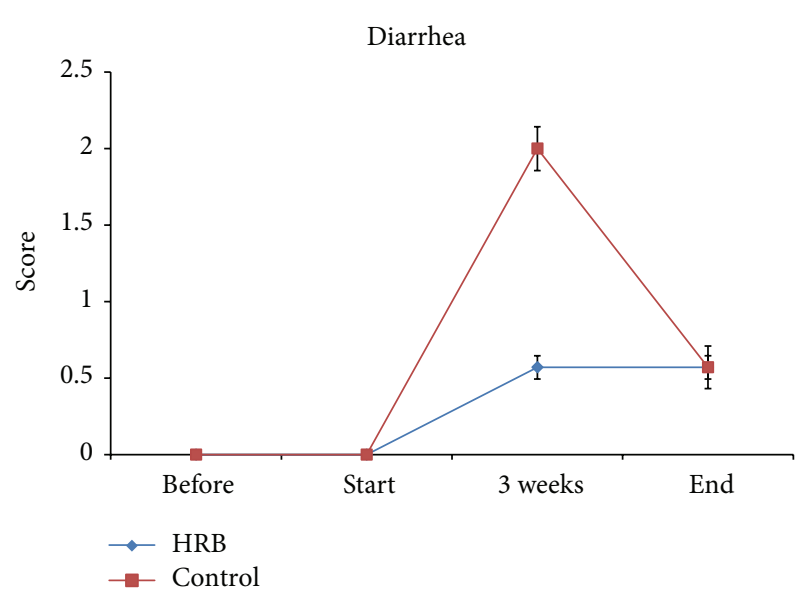

(a)

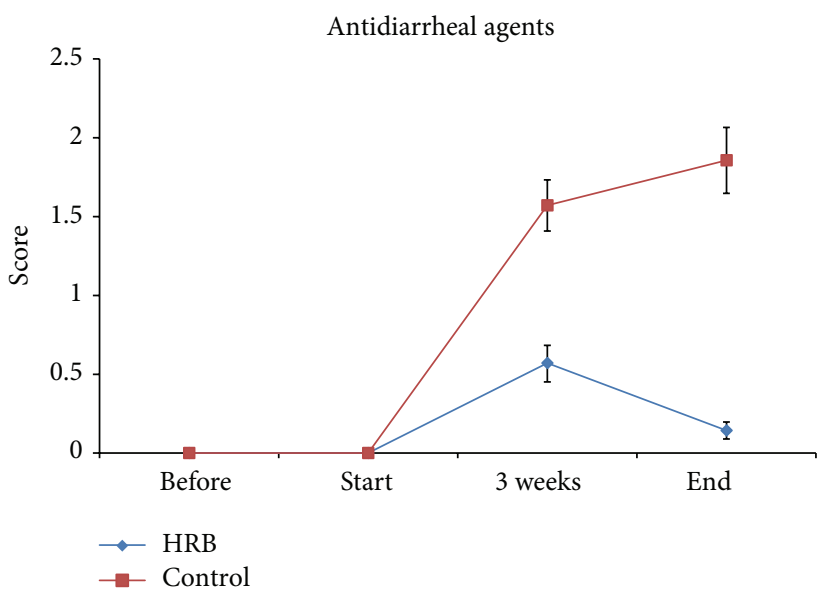

(b)

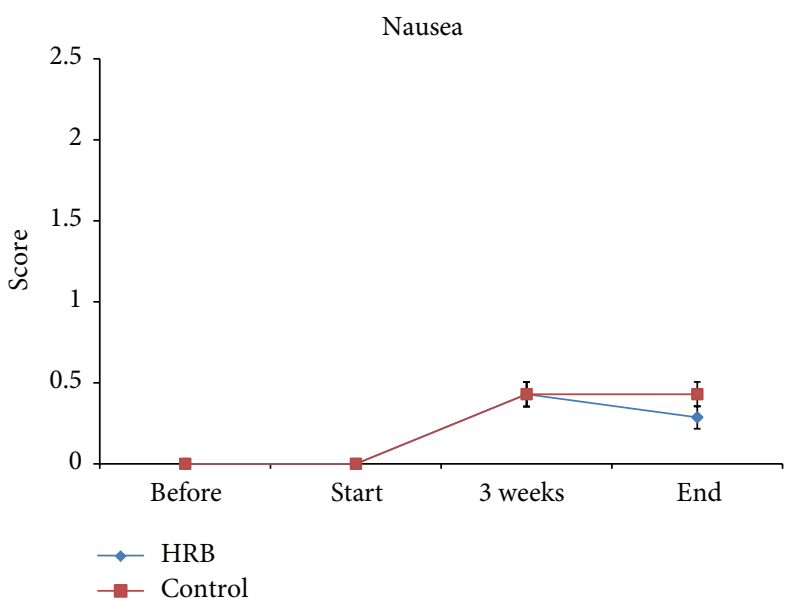

(c)

FIGURE 1: (a) Effects of HRB on diarrhea after chemoradiotherapy. (b) Effects of HRB on use of antidiarrheal agents after chemoradiotherapy. (c) Effects of HRB on nausea after chemoradiotherapy.

were squamous cell carcinoma in 18 patients and adenosquamous carcinoma in 2 . In all 20 patients with cervical cancer, treatment began with chemoradiotherapy, but, in 2 of the 20 patients, the radiation therapy was discontinued about 1 month after it was started, and switched to surgery. These two patients were therefore excluded from the assessments. We also excluded another four patients in whom the ingestion rate of the test food was low (ingestion rate of $0 \%$ at both 3 weeks and at completion; all of these patients had strong nausea and vomiting due to the chemoradiotherapy and consumed almost nothing by mouth). After excluding these 6 patients, we assessed the results of 14 patients (HRB group, 7 patients; control group, 7 patients). The frequency and severity of diarrhea, the primary endpoint, were not significantly different between the HRB and control groups in the third week of radiation therapy, but the diarrheal side effect assessment score was lower in the HRB group, and a trend was seen toward a reduction in diarrhea symptoms with oral intake of HRB group (Figure 1(a)). There was also no significant difference in the administration of intestinal regulators and antidiarrheal agents, but again the assessment score was lower in the HRB group than in the control group, and diarrhea symptoms were alleviated with oral intake of HRB. A trend was observed toward no need for strong antidiarrheal agents (Figure 1(b)). No significant differences in nausea (Figure 1(c)), vomiting, or loss of appetite were seen between the HRB and control groups.

Similarly, no large difference in NK cell activity was seen between the two groups (Figure 2).

3.2. Safety Assessment. Blood test and serum chemistry results were compared between before and at the end of consumption of the test food. The white blood cell counts before and after the treatment are shown in Table 2. Patients with high values were seen in both the HRB and control groups, but in all of these patients, the high counts were judged to be an inflammatory response to the cancer (most were pyometra). The white blood cell counts were also significantly lower in both groups at the end of chemoradiotherapy, but this was considered to be mainly a side effect of the chemoradiotherapy.

There was no significant interaction between the two groups in the decrease of the white blood cell count. However, five of seven patients in the HRB group showed normal white 


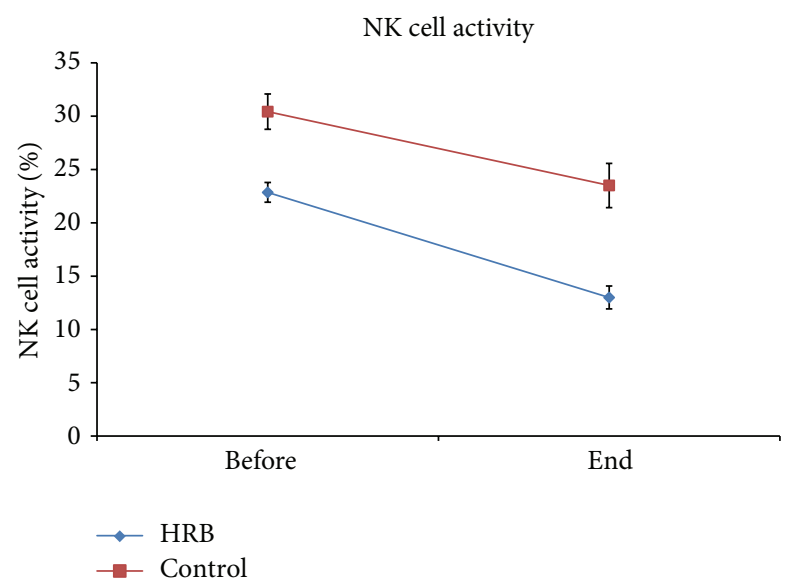

FIgURE 2: NK cell activity before and after chemoradiotherapy.

TABLE 2: White blood cell counts before and after treatment.

\begin{tabular}{ccccc}
\hline & \multirow{2}{*}{ Age } & \multicolumn{2}{c}{ WBC (cells/ $\mu \mathrm{L})$} & \multirow{2}{*}{ Grade (after) } \\
& 64 & 8700 & 2500 & 2 \\
& 40 & 10000 & 3700 & 0 \\
HRB & 70 & 7700 & 3400 & 0 \\
& 62 & 10300 & 5600 & 0 \\
& 44 & 7000 & 4000 & 0 \\
& 31 & 8900 & 2600 & 2 \\
& 38 & 6400 & 5700 & 0 \\
\hline \multirow{4}{*}{ Control } & 72 & 6300 & 3200 & 1 \\
& 58 & 8200 & 3400 & 0 \\
& 69 & 10200 & 4600 & 0 \\
& 51 & 10200 & 2000 & 2 \\
& 52 & 7900 & 1600 & 3 \\
& 40 & 11000 & 2400 & 2 \\
\hline
\end{tabular}

blood cell counts, whereas three of seven patients in the control group showed normal white blood cell counts. When expressed by National Cancer Institute grading, two patients in the HRB group developed grade 2 adverse events, while one, two, and one patient in the control group developed grade 1,2, and 3 adverse events, respectively. The control group tended to develop adverse events of higher grades than did the HRB group. Furthermore, when the grade was the same, the white blood cell counts tended to be lower in the control group (Table 2).

No marked differences were seen in the other items between the HRB and control groups. Observed side effects (adverse events) were hypokalemia in one patient in the HRB group and cystitis in one patient in the control group. In both cases, however, the physicians diagnosed the side effects as unrelated to consumption of the test food.

\section{Discussion}

HRB is a processed food, the basic ingredient of which is water-soluble rice bran fiber. In this study, we paid attention to the colitis-inhibiting action of HRB and conducted an exploratory investigation of its effect in relieving acutephase gastrointestinal side effects of chemoradiotherapy for cervical cancer. We set the frequency and severity of diarrhea symptoms as the primary endpoint, and the frequency and severity of gastrointestinal symptoms other than diarrhea (nausea, vomiting, and loss of appetite) as the secondary endpoint. However, because these items are affected by patients' subjective symptoms, we performed a prospective, randomized, placebo-controlled, double-blind study for a more rigorous assessment of the effects of HRB. However, the sample size did not have enough statistical power because this study was an exploratory study to determine the efficacy of the test food, serve as a baseline to determine the sample size of future detailed studies, and gather information.

In this study, no significant difference was detected in the primary and secondary endpoints between the two groups. However, because diarrhea, a side effect of chemoradiotherapy, in the HRB group showed an inclination to be less frequent than that in the control group, oral intake of HRB may enable a reduction in the dose of drugs generally administered to treat diarrhea and other gastrointestinal side effects in the acute phase $[8,9]$. This could make it possible to continue treatment while maintaining patients' quality of life during the treatment. There was no significant difference in the decrease of NK cell activity before and after intake of the test food between the HRB and control groups. However, decreases in the white blood cell count were seen in only two of seven patients in the HRB group, whereas such decreases were seen in four of seven patients in the control group. With respect to adverse events, only two patients developed grade 2 adverse events in the HRB group, while one, two, and one patient in the control group developed grade 1, 2, and 3 adverse events, respectively. Thus, more severe side effects occurred in the control group. Furthermore, when both groups exhibited the same grade 2 adverse events, the white blood cell count itself tended to be lower in the control group (Table 2).

This study was an exploratory clinical trial conducted as a pilot study with a small number of patients. However, the results suggest that HRB may relieve diarrhea, an acutephase gastrointestinal side effect of chemoradiotherapy for cervical cancer, and may inhibit the decrease in white blood cells that occurs with chemoradiotherapy. Additionally, no safety problems were observed in this study. While there were no significant differences between the two groups, the results seem promising with respect to the efficacy of HRB in reducing diarrhea symptoms and inhibiting a decrease in the white blood cell count. A multicenter, large-scale prospective clinical trial will be needed to confirm these benefits of HRB intake.

\section{Conclusion}

Our study suggested that HRB may relieve diarrhea, an acute-phase gastrointestinal side effect of chemoradiotherapy in patients with cervical cancer, although this randomized, double-blind study is exploratory and also a sample size is very small. 


\section{Conflict of Interests}

The authors declared that they had no conflict of interests.

\section{Authors' Contribution}

Yoshiyuki Itoh, Mitsuru Ikeda, and Mika Mizuno were responsible for the conception and design. Yoshiyuki Itoh, Mitsuru Ikeda, Mika Mizuno, Rie Nakahara, Seiji Kubota, and Junji Ito were responsible for collection and assembly of data. Yoshiyuki Itoh, Mitsuru Ikeda, and Mika Mizuno were responsible for data analysis and interpretation. All authors were responsible for paper writing and final approval of paper.

\section{Acknowledgment}

The authors deeply appreciate Daiwa Pharmaceutical Co., Ltd., the manufacturer of both the HRB and placebo foods, which were provided free of charge.

\section{References}

[1] H. M. Keys, B. N. Bundy, F. B. Stehman et al., "Cisplatin, radiation, and adjuvant hysterectomy compared with radiation and adjuvant hysterectomy for bulky stage IB cervical carcinoma," The New England Journal of Medicine, vol. 340, no. 15, pp. 1154$1161,1999$.

[2] M. Morris, P. J. Eifel, J. Lu et al., "Pelvic radiation with concurrent chemotherapy compared with pelvic and para-aortic radiation for high-risk cervical cancer," The New England Journal of Medicine, vol. 340, no. 15, pp. 1137-1143, 1999.

[3] P. G. Rose, B. N. Bundy, E. B. Watkins et al., "Concurrent cisplatin-based radiotherapy and chemotherapy for locally advanced cervical cancer," The New England Journal of Medicine, vol. 340, no. 15, pp. 1144-1153, 1999.

[4] J. A. Green, J. M. Kirwan, J. F. Tierney et al., "Survival and recurrence after concomitant chemotherapy and radiotherapy for cancer of the uterine cervix: a systematic review and metaanalysis," The Lancet, vol. 358, no. 9284, pp. 781-786, 2001.

[5] L. Portelance, K. S. C. C. Chao, P. W. Grigsby, H. Bennet, and D. Low, "Intensity-modulated radiation therapy (IMRT) reduces small bowel, rectum, and bladder doses in patients with cervical cancer receiving pelvic and para-aortic irradiation," International Journal of Radiation Oncology Biology Physics, vol. 51, no. 1, pp. 261-266, 2001.

[6] J. M. Kirwan, P. Symonds, J. A. Green, J. Tierney, M. Collingwood, and C. J. Williams, "A systematic review of acute and late toxicity of concomitant chemoradiation for cervical cancer," Radiotherapy and Oncology, vol. 68, no. 3, pp. 217-226, 2003.

[7] A. J. Mundt, A. E. Lujan, J. Rotmensch et al., "Intensitymodulated whole pelvic radiotherapy in women with gynecologic malignancies," International Journal of Radiation Oncology Biology Physics, vol. 52, no. 5, pp. 1330-1337, 2002.

[8] A. B. Benson III, J. A. Ajani, R. B. Catalano et al., "Recommended guidelines for the treatment of cancer treatmentinduced diarrhea," Journal of Clinical Oncology, vol. 22, no. 14, pp. 2918-2926, 2004.

[9] R. J. Gibson, D. M. K. Keefe, E. B. Lalla et al., "For the mucositis study group of the multinational association of supportive care in cancer/international society of oral oncology
(MASCC/ISOO). Systemic review of agents for the management of gastrointesitinal mucositis in cancer patients," Support Care in Cancer, vol. 21, pp. 313-326, 2013.

[10] Y. Hoshino, N. Hirashima, M. Nakanishi, and T. Furuno, "Inhibition of degranulation and cytokine production in bone marrow-derived mast cells by hydrolyzed rice bran," Inflammation Research, vol. 59, no. 8, pp. 615-625, 2010.

[11] K. Tazawa, K. Ichihashi, T. Fujie, K. Omura, M. Anazawa, and H. Maeda, "The orally administration of the hydrolysis rice bran prevents a common cold syndrome for the elderly people based on immnunomodulatory function," Journal of Traditional Medicines, vol. 20, pp. 132-141, 2003.

[12] M. Ghoneum and S. Abedi, "Enhancement of natural killer cell activity of aged mice by modified arabinoxylan rice bran (MGN-3/Biobran)," Journal of Pharmacy and Pharmacology, vol. 56, no. 12, pp. 1581-1588, 2004.

[13] J. T. Thornthwaite, H. Shah, P. Shah, and H. Respess, "The natural killer cell: a historical perspective and the use of supplements to enhance NKC activity," Journal of Immune Based Therapies, Vaccines and Antimicrobials, vol. 1, no. 3, pp. 21-51, 2012. 


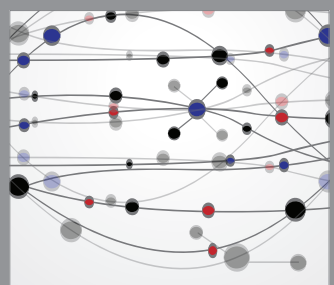

The Scientific World Journal
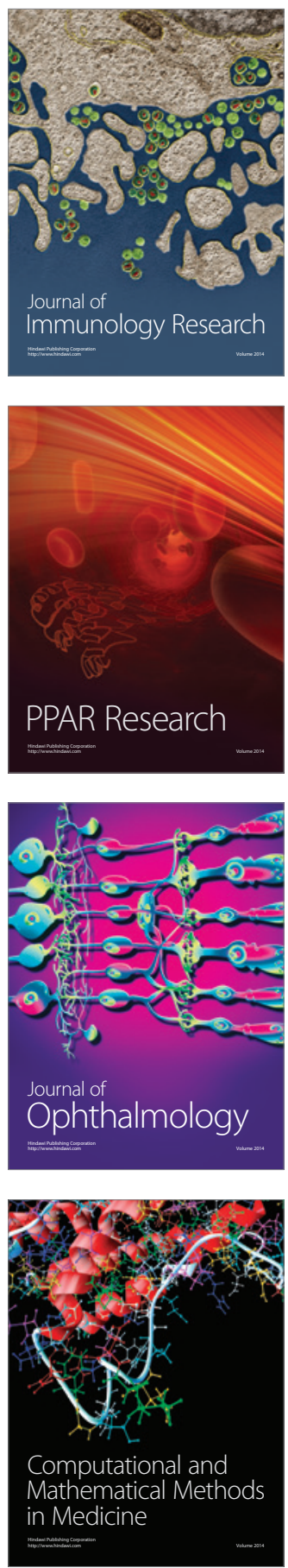

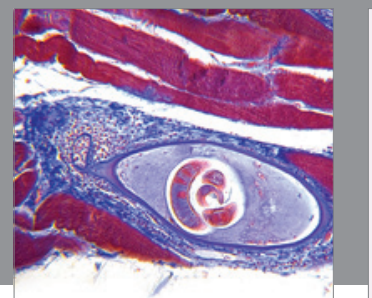

Gastroenterology

Research and Practice
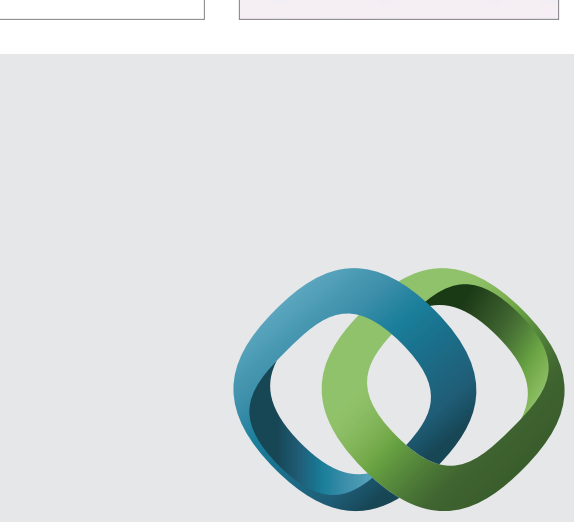

\section{Hindawi}

Submit your manuscripts at

http://www.hindawi.com
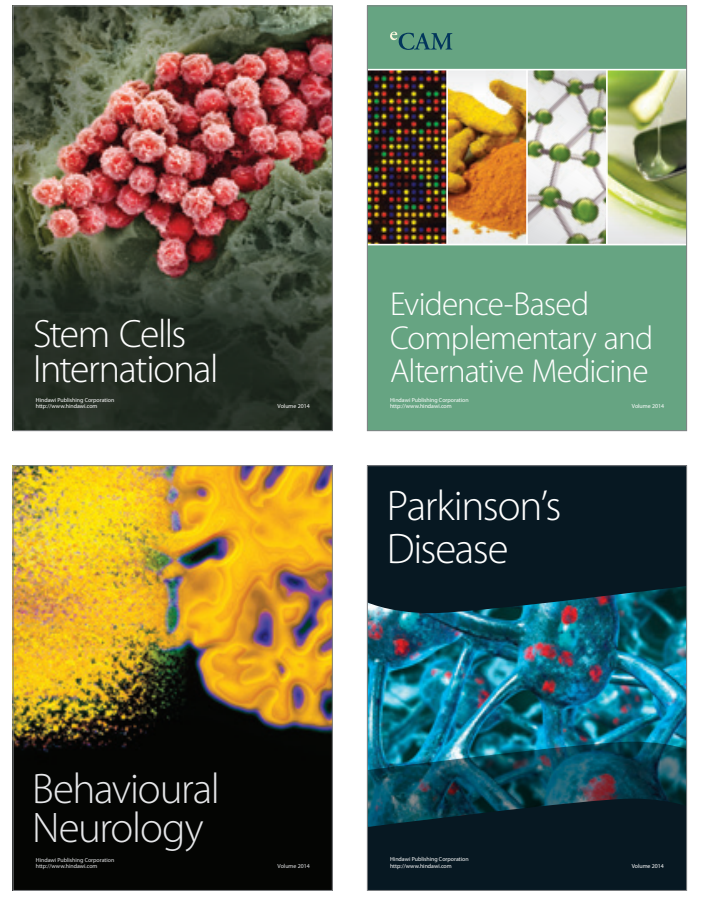
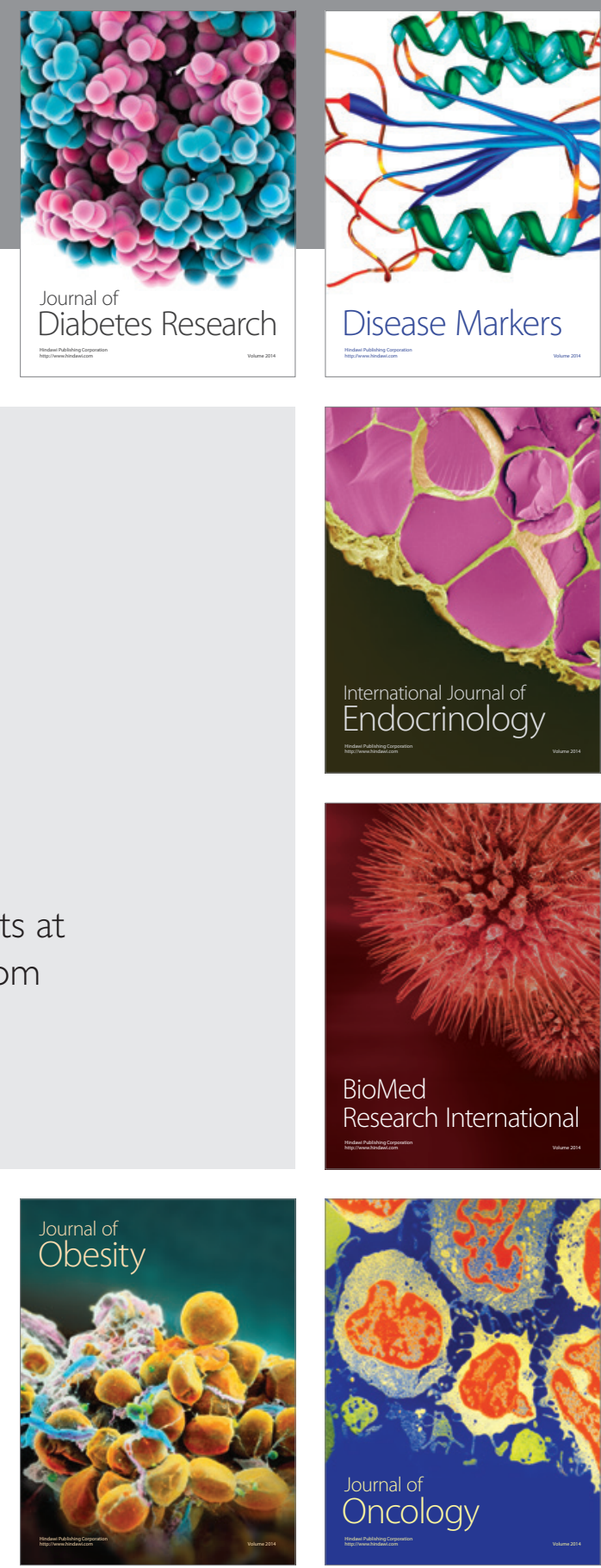

Disease Markers
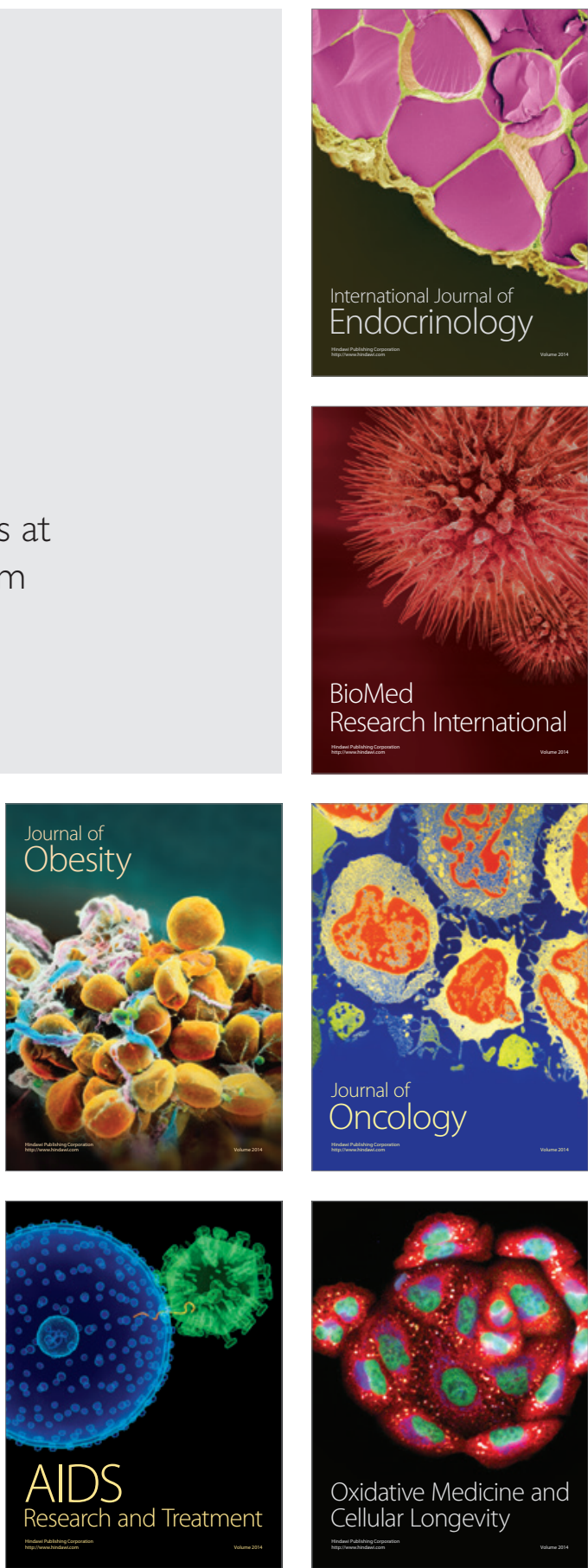